\title{
Empoderamiento: Una Caracterización al Interior de la Educación Matemática
}

\author{
Empowerment: A Characterization within Mathematics \\ Education
}

\section{Empoeiramento: Uma Caracterização em Educação Matemática}

\author{
Adriana N. Magallanes *1 \\ Cristina B. Esteley ${ }^{2}$ \\ ${ }^{1}$ Universidad Nacional de Río Cuarto ${ }^{2}$ Universidad Nacional de Córdoba
}

\begin{abstract}
El presente trabajo pone en juego un proceso analítico-constructivo con el fin de realizar una caracterización del empoderamiento para el ámbito de la educación matemática. Para llegar a tal caracterización se delimitan algunos supuestos e ideas en torno al empoderamiento haciendo un recorrido de discusiones acerca de esta noción desde una perspectiva amplia. Se discuten posibles interacciones o vínculos entre ella para darle sentido al empoderamiento en el ámbito educativo en general y en el campo de la educación matemática en particular. Al interior de la educación matemática, se recuperan aportes de autores reconocidos en el campo y se propone una caracterización del empoderamiento que da cuenta de tres dimensiones: matemática, social y epistemológica. Con ello, se recuperan las ideas de participación democrática y trabajo autónomo, entre otras. La caracterización propuesta adquiere relevancia en situaciones de trabajo de producción de modelos matemáticos en clases de matemática.
\end{abstract}

Descriptores: Empoderamiento, Educación matemática, Modelización matemática, Participación democrática.

\begin{abstract}
This work brings into play an analytical-constructive process in order to make a characterization of empowerment for the field of mathematics education. In order to reach such a characterization some assumptions and ideas about empowerment are discussed from a broad point of view. Then, we propose interactions among those ideas in order to make sense of empowerment within the educational sphere in general and within the field of mathematics education in particular. Within mathematics education, we recover contributions from recognized authors in the field and we propose a characterization of empowerment that accounts for three dimensions: mathematical, social and epistemological. With this characterization we intend to regain the ideas of democratic participation and autonomy among others. Such characterization becomes relevant in situations of production work of mathematical models in math classes.
\end{abstract}

Keywords: Empowerment, Mathematics education, Mathematical modelling, Democratic participation.

\footnotetext{
*Contacto: adriana.n.magallanes@gmail.com $\quad$ Recibido: $\quad 17$ de marzo 2016

$1^{\text {a }}$ Evaluación: 22 de mayo 2016

ISSN: 2254-3139

$2^{\text {a }}$ Evaluación: 9 de agosto 2016

www.rinace.net/riejs/

Aceptado: $\quad 6$ de septiembre 2016
}

revistas.uam.es/riejs 
Este trabalho põe em jogo um processo de construção analítica, a fim de caracterizar a capacitação para o campo da educação matemática. Para alcançar tal caracterização algumas hipóteses e ideias sobre o empoeiramento fazendo um passeio de discussões sobre esta noção de uma perspectiva ampla entre-lo para fazer sentimento de capacitação em educação em geral e no campo da educação matemática, em particular, são discutidos. No âmbito da educação matemática, contribuições de autores de renome no campo são recuperados e uma caracterização de capacitação que realiza três dimensões é proposto: matemática, social e epistemológico. Com isso, as ideias de participação democrática e auto-emprego recuperar, entre outros. A proposta caracterização torna-se relevante no trabalho situações de produção de modelos matemáticos nas aulas de matemática.

Palavras-chave: Empoeiramento, Educação matemática, Modelagem matemática, Participação democrática.

\section{Introducción}

Este trabajo emerge en un proceso analítico-reflexivo en el marco de una investigación del campo de la educación matemática que busca dar cuenta de la existencia y características de procesos de empoderamiento en clases de matemáticas conformadas desde perspectivas compatibles con una Educación Matemática Crítica (Skovsmose, 2000) y el trabajo en aula con proyectos de modelización matemática (Esteley, 2014). En ese sentido, con este artículo se pretende poner en juego una reflexión crítica sobre la noción de empoderamiento o empowerment para luego focalizar la discusión sobre esta concepción en el ámbito educativo en general y en la educación matemática en particular. Finalmente y a partir de los aportes de diversos autores, así como desde nuestras propias experiencias como docentes e investigadoras, se propone una caracterización del empoderamiento para la educación matemática asumiendo la posibilidad de un vínculo sinérgico entre tales ideas y el trabajo con modelización matemática en las escuelas. Todo este proceso permite dar cuenta del objetivo planteado y pone en juego una actividad analítica-constructiva en la que se recuperan dialécticamente los aportes y las experiencias antes mencionados.

\section{Empoderamiento en un contexto amplio: estructuras descendentes y ascendentes}

A partir del análisis realizado, se puede indicar que actualmente coexisten numerosas definiciones y/o conceptualizaciones relacionadas con el empoderamiento, provenientes de diferentes campos y vinculadas con diferentes objetivos. Por ejemplo, desde el ámbito de la economía, Sen (1997) vincula empoderamiento con institución humana y con la libertad de las personas para alcanzar los objetivos de desarrollo, considerando capacidades que abarcan la vida, la salud y la integridad corporal, la capacidad de imaginar, reflexionar y razonar, la aptitud de exteriorizar los sentimientos, la razón práctica, la afiliación, el esparcimiento y el control sobre el entorno político y material de las personas. En un reporte del World Bank relativo a cuestiones de pobreza en el mundo, se considera el empoderamiento como un proceso que permite la construcción de los activos de los sujetos en situación de pobreza para que puedan participar de manera efectiva en los mercados (Ibrahim y Alkire, 2007). Como lo indica García Moreno (2005), en esta última conceptualización se observa un empoderamiento que se realiza desde una estructura de poder descendente donde los que controlan el dinero o ciertas 
condiciones deciden las reglas de juego y los propósitos de la acción, mientras que los destinatarios reciben lo que ha sido previamente concebido. En este sentido, se considera a los destinatarios como usuarios o beneficiarios que se empoderan participando de un conjunto de acciones, de tal modo que su participación posibilite el cumplimiento de metas y objetivos de los servicios que fueran elaborados de manera externa a los sujetos.

En la concepción propuesta por Sen (1997), se observa un empoderamiento que se realiza desde una estructura de poder ascendente (García Moreno, 2005). Esto es así pues se pretende ahondar en la democratización de la sociedad y la participación de las personas como ciudadanos y no como meros usuarios o beneficiarios. Desde tal perspectiva, se busca mejorar el flujo de poder ascendente mediante el cual las decisiones de orden público se toman consultando a los ciudadanos y tal vez por demanda de ellos mismos. Esta idea de poder ascendente alteraría las relaciones de poder ya establecidas y aceptadas como naturales.

Cabe indicar que, si bien las ideas de empoderamiento como estructuras ascendentes y descendentes parecen nociones dicotómicas, las mismas pueden ser pensadas como complementarias. Para comprender este aspecto, es importante reflexionar sobre los aspectos negativos y positivos del poder. Por ejemplo, si bien es necesario identificar el aspecto negativo del poder como fuerza de dominación y de represión, cabe señalar que esto no es suficiente para avanzar en una mayor comprensión del poder. De manera similar, reflexionar sobre el lado positivo del poder, como fuerza productiva, transformadora, creadora y de gran riqueza estratégica, tal vez tampoco es suficiente si se vincula únicamente a una estructura ascendente. Es importante notar que ambos aspectos del poder (negativo-positivo) pueden ser pensados tanto desde una estructura de poder descendente como ascendente. Desde esta perspectiva, se entiende que no es posible asociar el poder descendente como indeseable y el poder ascendente como bueno, ya que tanto uno como otro pueden ser ejercidos a favor o en contra de determinados sectores o grupos de la sociedad. Un poder descendente puede formular muy buenas intenciones a favor de un grupo particular, pero terminar por perjudicarlos. Por ejemplo, se pueden mencionar proyectos programados para mejorar la educación secundaria con objetivos, metas e incluso especificaciones en el gasto del presupuesto perfectamente definidas y estipuladas desde organismos centralizados (Ministerios de Educación provinciales o nacionales, entre otros organismos) que, al llegar a ciertas escuelas, no resuelven sus problemáticas específicas o más urgentes. Tal vez, para ilustrar, se puede citar el caso de escuelas de localidades pequeñas o aisladas a las que se les envió computadoras pero no disponían de suministro eléctrico. Tampoco se puede pensar que, por el solo hecho de que se esté en presencia de una estructura de poder ascendente, esto será suficiente para producir beneficios o cambios, ya que muchas veces será necesario que intervenga un poder descendente que tome decisiones, que destine presupuesto, que brinde condiciones para que los beneficios demandados puedan hacerse realidad. En este sentido, resulta más conveniente pensar y entender que lo que existe en la realidad son estructuras de poder donde interactúan de manera continua tanto el poder ascendente como el descendente, donde el poder ascendente será el encargado de cuidar que el poder descendente no imponga una sola verdad sino que atienda las distintas voces y genere oportunidades para que las voces silenciadas (Freire, 2006) puedan también ser escuchadas. Esta idea de interacción entre ambas estructuras o modelos se volverá a retomar en las conclusiones finales y luego de centrar nuestro análisis del empoderamiento en ámbitos educativos. 


\section{Reflexiones al interior del contexto educativo}

La filosofía del empoderamiento que se privilegia en esta sección tiene su origen en el enfoque de educación popular que se viene desarrollando desde la década de los 60, considerando al pedagogo brasileño Paulo Freire como principal impulsor. Freire realiza una distinción entre empowerment individual y social planteando que, si bien el empoderamiento individual es necesario, no es suficiente para un proceso de transformación social (Freire y Shor, 2014). La curiosidad, el pensamiento crítico y el "poder interno" al que alude Rowlands (1997) son fundamentales para la transformación de la sociedad, pero no son suficientes. Freire enfatiza que, cuando se persigue un cambio social tendiente a lograr mayores niveles de justicia social, se requiere considerar el social empowerment o empoderamiento social (Freire y Shor, 2014). Acorde a la categorización realizada por Rowlands (1997), el empoderamiento social se vincula con la idea de "poder con". Esto es así pues se entiende que el empoderamiento social se manifiesta cuando un grupo genera un espacio que posibilita que todos se expresen y desde el interior del grupo se formulen y construyan soluciones comunes para un problema definido y aceptado como tal por el grupo.

Tomando como base las ideas antes esbozadas y buscando profundizar las reflexiones hacia el interior del ámbito educativo con el interés de caracterizar la noción de empoderamiento, es importante analizar qué puede aportar tal caracterización a dicho ámbito, como así también es valioso reconocer los límites bajo los que se construye.

\subsection{Empoderamiento al interior del ámbito educativo: contribución, límites y caracterización}

En primer lugar, se debe pensar qué puede aportar para la educación llegar, o al menos aproximarnos, a una caracterización del empoderamiento. En una primer instancia y desde una perspectiva crítica, podemos señalar que tal caracterización puede contribuir a explicitar una visión considerada fundamental para la educación, así como ayudar a clarificar un horizonte esencial para orientar hacia dónde es conveniente dedicar esfuerzos para lograr una educación que contribuya a la conformación y el ejercicio de una ciudadanía democrática, no solo de los estudiantes sino también de los distintos actores educativos involucrados.

En segundo lugar, se explicitan los límites bajo los cuales se intenta conferir sentido al empoderamiento. Los límites se fijan particularmente, al menos en un principio, al interior del trabajo áulico, al trabajo cotidiano de docentes y estudiantes en un aula o espacio pensado para aprender y enseñar, para interactuar, para crear, para sorprender, para imaginar, para colaborar, para disfrutar del acto de aprender y enseñar. Brevemente, se habla de espacio para extender el sentido físico del aula a otros lugares tales como bibliotecas, espacios externos a la escuela como puede ser una salida de campo con fines investigativos junto a los estudiantes, un espacio virtual como puede ser un sitio web creado para interactuar, etc.

Es importante indicar que se considera el acto educativo como un proceso en el que tanto docente como estudiantes aprenden. El docente se mueve del lugar tradicional en el cual hace todas las preguntas y tiene todas las respuestas y, a partir de su formación y experiencia, actúa como guía del proceso, pero al mismo tiempo está abierto para recuperar las voces de los estudiantes, así como también está dispuesto a generar 
condiciones para que esas voces puedan surgir y para que las preguntas e inquietudes de los estudiantes encuentren un espacio donde puedan ser expresadas y atendidas.

Acotando los límites bajo los que se pretende caracterizar empoderamientos y considerando las contribuciones antes indicadas que podría traer tal caracterización, avanzamos hacia ella tomando en un primer momento aportes de Ernest (2002). Las ideas de este autor resultan relevantes y básicas para nuestra caracterización, del mismo modo que lo es señalar que es el modo como se lleve a cabo el acto educativo lo que permitirá el empoderamiento y que, en tal caso, tanto estudiantes como docentes serán empoderados gracias a este proceso.

Con estas ideas, de ninguna manera se entiende que el docente deba desplazarse de su rol y dejar en el alumno u otro actor las responsabilidades que le competen; simplemente se quiere explicitar de qué manera se entiende ese rol y que la búsqueda esencial del docente será la de tratar de generar condiciones para que tenga lugar este proceso de empoderamiento.

Torres (2009) va más allá de una mera caracterización y define empoderamiento como el proceso de concienciación que da cuenta al estudiante de sus capacidades desde lo cual potencia su acción para transformarse y transformar su contexto. Si bien esta definición resulta de interés, para este trabajo, que focaliza en el aula o en el espacio (en el sentido antes mencionado) donde se produce el acto educativo, es importante indicar que la misma no aclara cómo el proceso de empoderamiento puede producir la concienciación de las capacidades del estudiante para potenciar su acción o para aumentar su autoestima.

En el video "Pedagogía de la Esperanza, Freire Paulo - Fragmentos y reflexiones1", desde el minuto 5:50 al minuto 6:40, se pone en evidencia, de una manera simple, lo que se considera uno de los principales factores de la "pedagogía del aburrido" (Corea y Lewkowicz, 2004). Tal pedagogía es compatible con una educación basada en preguntas que solo hace el docente y a las que se espera que el alumno responda sabiendo que el docente ya conoce las respuestas y que a él solo le compete acercarse con su respuesta a la del docente. Este tipo de interacciones parecen ser uno de los aspectos que quitarían el entusiasmo y la motivación en el alumno por el acto de aprender, ya que no solo la pregunta puede no ser "su pregunta", sino que, además, el esfuerzo por la búsqueda en responder no tiene demasiado sentido, pues se sabe desde el principio que la respuesta ya es conocida por su maestro. Este acto pedagógico puede ser visto como el paso de un acto educativo a un acto de control. Se sugiere que, desde una perspectiva crítica, es esencial moverse de una pedagogía de la respuesta a una pedagogía de la pregunta en el sentido de Freire (1986). Planteamos así que el empoderamiento en el ámbito educativo debería ser un proceso de interacción entre docentes y estudiantes que les permita tomar conciencia de sus capacidades para tomar decisiones, para actuar no solo de manera individual sino colectiva, para transformarse y transformar su contexto. Pero, para que esto sea posible, es necesario que este proceso:

- De lugar o abra un espacio para el pensamiento y las preguntas de los estudiantes (no solo de los docentes), antes que un espacio para la repetición o búsqueda de una o varias respuestas correctas. Lo esencial no es que el

${ }^{1}$ https://www.youtube.com/watch?v=GjKWoP6uV2o\#aid=P92HYDPfeZY 
estudiante sea un depositario de información y conocimientos, sino que pueda buscar esa información, que pueda ejercitar su pensamiento y, a partir de ello, pueda hacerse sus propias preguntas, buscar otros conocimientos y otra información que le ayude a encontrar respuestas a tales preguntas.

- Tenga en cuenta que en la búsqueda de información y conocimientos, así como en la formulación de preguntas, se atribuye sentido a su trabajo si el punto de partida es la indagación de alguna problemática que resulta cercana o de interés del estudiante. Nadie se involucra y nadie aprende sobre algo que no despierta su interés o algo que no le ayude a responder alguna pregunta que es o siente como propia.

- Permita, ante preguntas compartidas, abrir un espacio para la indagación y la toma de decisiones colectivas, para la construcción colectiva de respuestas, para escuchar, ser críticos y autocríticos de los caminos de respuestas escogidos, existiendo en todo momento apertura para realizar cambios o modificaciones en esas respuestas (incluyendo en esta afirmación las decisiones y respuestas del docente).

Estos puntos podrían contribuir a sostener una educación que pretenda generar en el estudiante el "gusto por aprender" y, más aún, una educación que no ponga el énfasis en la mera acumulación de contenidos sino en abrir un espacio donde el estudiante tenga la posibilidad de hacer sus preguntas, escuchar las preguntas de otros y aventurarse en una búsqueda colectiva donde cada uno desde su lugar, desde su formación y experiencia, pueda hacer aportes; donde todos puedan reconocer que lo importante no es solo conocer o comprender un concepto en particular, sino que lo importante es cómo el modo de construir y comprender ese concepto les permite al docente y al estudiante sentirse empoderados. El docente puede percibir que su labor tiene sentido, que puede servirle al estudiante y que él mismo vuelve a sentir el gusto por enseñar aprendiendo y aprender enseñando. El alumno puede reconocer que aprender tiene sentido, que puede hacer sus preguntas y que estas preguntas pueden servir para construir su propio proyecto educativo, atendiendo a un proyecto colectivo y donde la posibilidad de servir a la sociedad y a su propio contex to otorgue un nuevo sentido al acto educativo.

Las ideas recién presentadas son compatibles en cierto sentido con lo discutido en Rowlands (1997), quien considera tres categorías relativas al poder: poder sobre, poder para y poder con.

El poder sobre se evidencia cuando el poder de un sujeto o grupo significa una pérdida de poder del otro u otros. Por ejemplo, en nuestros actuales ámbitos educativos, es común que algunos docentes perciban pérdida de poder conjuntamente con un incremento en el poder del alumno, considerando este hecho como una disminución del poder que años atrás implicaba su presencia en el aula. Ante esta percepción, muchas veces lo que se intenta hacer es endurecer los mecanismos de control y de sumisión para lograr recuperar ese poder sobre. Esta vivencia puede ser reflexionada desde otro tipo de mirada en la cual se pueda pensar que el alumno está cuestionando los patrones de poder que históricamente han existido en las escuelas, no solo sobre las ideas, creencias y valores, sino también sobre los cuerpos y sobre las actitudes de los alumnos, quienes debían ser disciplinados en su tránsito por las escuelas. Además, este disciplinamiento se debía hacer según lo establecido por los docentes, las autoridades escolares y/o las 
políticas educativas del gobierno de turno, enfatizando de esta manera una estructura de poder descendente. Esta observación puede hacer pensar de otra manera lo que hoy se está viviendo en las escuelas y puede ser una mirada que ayude a pensar en otras estrategias para abordar la problemática en las cuales el docente no se quede aferrado a querer imponerse o lograr la sumisión de parte de los alumnos, sino que se pueda pensar que el desafío para el docente consiste en:

- Asumir que, aunque es el poseedor de una posición dominante de manera formal, esta no es suficiente hoy para garantizar la aceptación de la misma por parte de los estudiantes ni de las familias.

- Cuestionar los patrones de poder que siempre se han aceptado en la educación como naturales y que, aunque pueden haber contribuido al orden y a la sumisión por muchas décadas, lo que hoy es posible y necesario que los estudiantes logren en su tránsito por las escuelas no puede estar focalizado en este tipo de valores. Es necesario empezar a pensar en otros valores que logren empoderarlos más que domesticarlos, que les permitan tomar consciencia acerca de su potencial para beneficiar la convivencia en las aulas y en las escuelas, así como para ser reflexivos y activos en una educación que habilite espacios para mejorar su calidad de vida y la de su entorno.

El poder para es aquel poder que tienen algunas personas para estimular la actividad de otras, para generar posibilidades de acción en otro sin que exista dominación. Hoy es muy difícil que sean docentes o autoridades educativas los que puedan generar o mejorar la convivencia en las aulas, mejorar la calidad de vida de los alumnos o su entorno, etc., sin la colaboración de estudiantes, familias u otros agentes. Se busca, en este caso, una auténtica interacción de estructuras ascendentes y descendentes como medio que da fortaleza. Se plantea que este poder para solo puede estar presente si se da conjuntamente con un poder con.

El poder con se manifiesta cuando un grupo genera una solución colectiva para un problema común, problema que parte desde el interior del grupo y que posibilita que todos se expresen, tanto en la formulación de las problemáticas como en la construcción de una solución.

El poder interno o desde adentro se basa en la generación de confianza en uno mismo, se encuentra muy relacionado con la autoestima y se manifiesta en la habilidad para resistir al poder de otros, rechazando demandas no deseadas o exigiendo demandas propias. A partir de las observaciones anteriores, se entiende que este poder interno (tanto de estudiantes como de docentes) será una de las consecuencias de cambios cotidianos en el aula orientados por esta visión en el campo educativo. Se sabrá que se está transitando por un camino del empoderamiento cuando docentes y estudiantes reconozcan que todos tienen la posibilidad de influir y de actuar para cambiar sus problemáticas en el ámbito educativo.

Finalmente, para que la actividad educativa pueda producir el empoderamiento de docentes y estudiantes, debe dar lugar al pensamiento y la formulación de preguntas por parte de todos y a que, en este espacio de preguntas compartidas, se abra el espacio para la indagación y la toma de decisiones colectivas, para la construcción colectiva de respuestas, para escuchar a los otros y ser críticos y autocríticos de los caminos de 
respuestas escogidos y estar siempre abiertos a realizar cambios o modificaciones en sus respuestas.

Se considera relevante plantear esta última reflexión, ya que la modelización a la cual se alude en la educación matemática puede ser también realizada desde el paradigma de la respuesta, en cuyo caso solo se estaría realizando una modificación que puede llegar a hacer un poco más atractivo y/o lograr "cierta razón de ser" (Chevallard, 1999) del conocimiento, pero que difícilmente logre empoderar a docentes, ni mucho menos a los estudiantes que interactúen en dicha práctica educativa.

\subsection{Empoderamiento al interior del ámbito de la educación matemática}

Entendiendo el empoderamiento como un proceso a través del cual tanto docentes como estudiantes se empoderan, es valioso rescatar los aportes del educador matemático Paul Ernest (2002). Este autor destaca que, en procesos educativos que toman como referencia la enseñanza de la matemática, el empoderamiento se distribuye en tres dominios: el matemático, el social y el epistemológico. En el dominio matemático, el empoderamiento consiste en ganar poder sobre el lenguaje, los símbolos, las destrezas y la práctica de usar y aplicar el conocimiento específico de la matemática, en la actividad de "hacer matemática". En la dimensión social, el empoderamiento significa que docentes y estudiantes puedan tomar conciencia tanto de la naturaleza de la matemática y sus aplicaciones como así también de la matemática como una herramienta de pensamiento para ver el mundo, comprenderlo y criticarlo, contribuyendo al poder sobre el ámbito político y social y, posiblemente, a la promoción de la justicia social y a una mejor forma de vida para todos en entornos próximos o más distantes. Finalmente, en relación con la dimensión epistemológica, el empoderamiento se refiere al crecimiento en la confianza no solo en el uso de la matemática sino también en el poder personal sobre la creación del conocimiento y la validación del mismo en el contexto de producción.

Para avanzar en el sentido de tales dimensiones es importante explicitar que, adhiriéndose a la perspectiva discutida en Devlin (1994), se considera la matemática como la ciencia de los modelos. Desde este enfoque, sobre la matemática podemos pensar en proyectos de modelización matemática para el aula y repensar las dimensiones propuestas por Ernest como el propio proceso de empoderamiento. Para dar sentido a este hecho, podríamos indicar, muy brevemente, que al hablar de "proyectos de modelización matemática para el aula” estamos pensando en un ambiente educativo en el cual los estudiantes, como grupo, escogen un fenómeno (intra o extramatemático) de su interés para estudiar, plantean problemas relacionados con dicho fenómeno, seleccionan variables, levantan hipótesis, diseñan experimentos (si es necesario), buscan información, recolectan y realizan tratamiento de datos, resuelven el problema, abren instancias de validación del trabajo, escriben un reporte y comunican sus resultados.

Esta visión tiene fuertes implicancias en la problemática de la enseñanza de la matemática que comienzan a gestarse desde los 80 en diversos países europeos y en Brasil. A comienzos de los 2000 se hacen presentes en Argentina algunas experiencias áulicas relacionadas con la enseñanza de la matemática en escenarios de modelización (Esteley, 2014). Algunos resultados comienzan a enfatizar las dificultades e incertidumbre que genera al profesor la puesta en aula de la modelización como estrategia pedagógica que enfatiza la generación de modelos a partir de problemas seleccionados por alumnos como así también los nuevos roles que comienzan a tener los estudiantes (Barbosa, 2001; Esteley, 2014; Villarreal, Esteley, Mina y Smith, 2010; 
Villarreal, Esteley y Smith, 2011). Ello es así pues la actividad de modelización, presente en los actuales Diseños Curriculares para la Provincia de Córdoba, implica apelar a una visión dinámica de la matemática que permite establecer interconexiones entre la matemática y otras áreas de conocimiento o entre diferentes áreas de la propia matemática, superando una visión centrada en la mera aplicación de modelos matemáticos ya construidos por otros. Como lo señalan Esteley (2014) o Barbosa (2001), entre otros, los condicionamientos institucionales producen también un cierto grado de incertidumbre al momento de decidir la puesta en aula de este particular escenario. Este hecho pondría en evidencia, en el marco de una institución educativa particular, una interacción entre estructuras de poder descendente (de la institución hacia el interior del aula o espacio) y una estructura de poder ascendente en el otro sentido de la relación.

De manera similar se evidencia tal interacción entre institución escolar y ministerios de educación tomando en este caso como instrumento los Diseños Curriculares.

Las ideas brevemente discutidas en las dos últimas secciones dan un marco para aproximarnos a una caracterización de empoderamiento en el ámbito de la educación matemática.

\section{Conclusiones: una caracterización de empoderamiento matemático}

Posicionarse desde una perspectiva de búsqueda de una caracterización del empoderamiento en el campo de la educación matemática implica ser cautos en esa búsqueda tratando de que la misma sea lo suficientemente amplia pero también reconociendo que finalmente uno se posiciona. En este sentido el posicionamiento se hace evidente en instancias de la caracterización, lo que implica entre otras cuestiones un posicionamiento relativo al trabajo matemático en las aulas.

En esta última cuestión es importante indicar que partimos en primera instancia de una búsqueda de contribuir para que los alumnos aprendan a través o por medio de la matemática y no solo matemática. En esta perspectiva tan amplia, trabajar con "proyectos de modelización matemática para el aula" es aceptado como potenciador de aquella idea y como un medio que entra en sinergia con una idea de empoderamiento que busca no solo una circulación de poder con, poder para y poder interno (en el sentido de Rowlands, 1997) sino que además se requiere que docente y estudiantes acepten el desafío de trabajar con proyectos de modelización matemática en el sentido antes explicitado, con la incertidumbre que produce ese tipo de trabajo.

En ese contexto, los dominios de empoderamiento propuestos por Ernest (2002) pueden ser reformulados de modo tal que:

En el dominio matemático, el empoderamiento consiste en ganar poder para seleccionar fenómenos (intra o extra matemáticos), para formular un problema a estudiar, para generar conjeturas, validarlas o refutarlas y generar condiciones para buscar soluciones, en todos los casos, en interacción con otros actores. En ese proceso se va ganando también poder sobre el lenguaje, los símbolos, las destrezas y la práctica de usar, aplicar o generar conocimientos específicos de la matemática o fuera de la matemática en la actividad de "hacer matemática" en un espacio educativo. En la dimensión social, el empoderamiento significa que docentes, estudiantes u otros agentes puedan ir tomando 
conciencia tanto de la naturaleza de la matemática, sus aplicaciones y la naturaleza del fenómeno en estudio como así también de la matemática como una herramienta que posibilita conexiones con sus entornos próximos y de ese modo explorarlo, comprenderlo, criticarlo y, en última instancia, proponer ideas para buscar soluciones a lo criticado contribuyendo al poder sobre el ámbito político y social y, posiblemente, a la promoción de la justicia social y a una mejor forma de vida para todos quienes comparten entornos próximos o más distantes. Finalmente, en relación con la dimensión epistemológica, el empoderamiento se refiere al crecimiento en la confianza no solo en el uso de la matemática sino también en el poder personal sobre la creación del conocimiento y la validación del mismo en un contexto de producción matemática con una finalidad fijada por el grupo que acepte involucrarse en un proyecto de modelización y le dé sentido a una creación colectiva.

Esta caracterización guarda a su interior una participación democrática y crítica de los sujetos involucrados. Pretende fortalecer la autonomía en la toma de decisiones y acciones de estudiantes y docentes en el marco de una visión renovada de la enseñanza de la matemática que busca poner en juego un ambiente investigativo con referencia en la realidad para ir más allá de un ambiente de aprendizaje centrado en el mero ejercicio matemático o de aplicaciones en contextos de semi-realidad (Skovsmose, 2000).

\section{Agradecimiento}

Se agradece al Dr. Eduardo Sota (Universidad Nacional de Córdoba) por haber inspirado la discusión sobre empoderamiento en un sentido amplio.

\section{Referencias}

Barbosa, J. (2001). Modelagem matemática: Concepções e experiências de futuros professors (Tesis doctoral). Universidade Estadual Paulista, Rio Claro, Brasil.

Chevallard, Y. (1999). L'analyse des pratiques enseignantes en théorie anthropologique du didactique. Recherches en Didactique des Mathématiques, 19(2), 221-266.

Corea, C. y Lewkowicz, I. (2004). Pedagogía del aburrido: escuelas destituidas, familias perplejas. Buenos Aires: Paidós.

Devlin, K. (1994). Mathematics: the science of patterns. Nueva York: Scientific American Library.

Ernest, P. (2002). Empowerment in mathematics education. Philosophy of Mathematics Education Journal, 15(1), 1-16.

Esteley, C. (2014). Desarrollo profesional en escenarios de modelización matemática: voces y sentidos. Córdoba: Universidad Nacional de Córdoba.

Freire, P. (1986). Hacia una pedagogía de la pregunta. Conversaciones con Antonio Faúndez. Buenos Aires: Autor.

Freire, P. (2006). Pedagogía de la autonomía: saberes necesarios para la práctica educativa. Buenos Aires: Siglo Veintiuno Editores Argentina.

Freire, P. y Shor, I. (2014). Miedo y osadía: la cotidianidad del docente que se arriesga a practicar una pedagogía transformadora. Buenos Aires: Siglo Veintiuno Editores Argentina. 
García Moreno, M. (2005). Los tres reinos del empoderamiento: Ambigüedad, contradicción e ilusión. En P. Crespo (Coord.), Empoderamiento: ¿tomar las riendas? (pp. 6-15). Quito: Secretaría Técnica ASOCAM.

Ibrahim, S. y Alkire, S. (2007). Agencia y empoderamiento en la medición de la pobreza. Revista Latinoamericana de Desarrollo Humano, 79, 1-7.

Rowlands, J. (1997). Questioning empowerment: working with women in Honduras. Oxford: Oxfam.

Sen, G. (1997). Empowerment as an approach to poverty. Working Paper Series, 97.07, 1-22.

Skovsmose, O. (2000). Escenarios de investigación. Revista EMA, 6(1), 3-26.

Torres, A. (2009). La educación para el empoderamiento y sus desafíos. Sapiens: Revista Universitaria de Investigación, 10(1), 89-108.

Villarreal, M., Esteley, C., Mina, M. y Smith, S. (2010). Mathematics teachers in modelling scenery: decisions while designing a project. Proceedings of the 34th Annual Conference of the International Group for the Psychology of Mathematics Education, 4, 273-280.

Villarreal, M., Esteley, C. y Smith, S. (junio, 2011). Desafíos y decisiones de profesores de matemática en escenarios de modelización: el diseño de un proyecto para el aula. Trabajo presentado en la XIII Conferencia Interamericana de Educación Matemática. Recife.

\section{Breve CV de las autoras}

\section{Adriana Noemi Magallanes}

Magíster en Didáctica de la Matemática (Universidad Nacional de Río Cuarto-UNRC); Técnico en Conducción educativa y Diplomado Superior en Ciencias Sociales (Facultad Latinoamericana de Ciencias Sociales, FLACSO); Profesora en Matemática (UNRC). Cursó posgrados en Educación y Sociedad; Poder, Autoridad y Educación; Investigación Educativa, Política Educativa; Filosofía y Sociología de la Educación entre otros. Se desempeñó como docente en el nivel medio, superior y universitario. Ocupó cargos de jefe de departamento y directora de nivel secundario. Participó como docente expositor en diversos congresos nacionales e internacionales y ha publicado artículos relacionados a Educación Matemática y Educación Estadística en diversas revistas locales y nacionales. Recibió distinciones a nivel nacional por su desempeño, trabajos educativos y de investigación. Actualmente se desempeña como Profesora e Investigadora de la Universidad Nacional de Río Cuarto. ORCID ID: 0000-0002-4404-6639. Email: adriana.n.magallanes@gmail.com

\section{Cristina B. Esteley}

Doctora en Educación, título otorgado por la Facultad de Filosofía y Humanidades de la Universidad Nacional de Córdoba (FFyH -UNC). Magíster en Educación Matemática, título otorgado por The City College de la City University of New York (Diploma de Honor). Profesora Ajdunta por concurso de antecedentes y oposición de la Facultad de Matemática, Astronomía, Física y Computación (FAMAF) -UNC. Dirige un grupo de investigación cuya principal temática de trabajo se vincula con la formación de profesores de matemática en un marco de trabajo colaborativo propiciando la elaboración de actividades centradas en procesos de modelización matemática. Una de las preocupaciones de su trabajo se centra en la búsqueda de medios para contribuir con una alfabetización matemática y tecnológica. Ha publicado libros, capítulos de libros y artículos en revistas nacionales e internacionales. Email: esteley@famaf.unc.edu.ar 